\title{
Rheological Characterization of Hydrogen Peroxide Gel Propellant
}

\author{
B.V.S. Jyoti* and Seung Wook Baek** \\ Division of Aerospace Engineering, School of Mechanical, Aerospace and Systems Engineering, Korea Advanced Institute of \\ Science and Technology (KAIST), Daejeon, Republic of Korea
}

\begin{abstract}
An experimental investigation on the rheological behavior of gelled hydrogen peroxide at different ambient temperature $(283.15,293.15$ and $303.15 \mathrm{~K})$ was carried out in this study. The gel propellant was rheologically characterized using a rheometer, in the shear rate ranges of 1 to $20 \mathrm{~s}^{-1}$, and 1 to $1000 \mathrm{~s}^{-1}$. Hydrogen peroxide gel was found to be thixotropic in nature. The apparent viscosity value with some yield stress (in-case of shear rate 1 to $20 \mathrm{~s}^{-1}$ ) drastically fell with the shear rate. In the case of the shear rate range of 1 to $20 \mathrm{~s}^{-1}$, the apparent viscosity and yield stress of gel were significantly reduced at higher ambient temperatures. In the case of the shear rate range of 1 to $1000 \mathrm{~s}^{-1}$, no significant effect of varying the ambient temperature on the gel apparent viscosity was observed. The up and down shear rate curves for hydrogen peroxide gel formed a hysteresis loop that showed no significant change with variation in temperature for both the 1 to $20 \mathrm{~s}^{-1}$ and the 1 to $1000 \mathrm{~s}^{-1}$ shear rate ranges. No significant change in the thixotropic index of gel was observed for different ambient temperatures, for both low and high shear rates. The gel in the 1 to $20 \mathrm{~s}^{-1}$ shear rate range did not lead to a complete breakdown of gel structure, in comparison to that in the 1 to $1000 \mathrm{~s}^{-1}$ shear rate range.
\end{abstract}

Key words: hydrogen peroxide, gel, shear thinning, thixotropy, rheological property

\section{Introduction}

Generally, gels are modified conventional liquids, whose properties are altered by the addition of a suitable gelling agent, so that their physico-chemical behavior undergoes a significant change [1-2]. Such a gel system must satisfy two criteria: the viscosity must be imparted by a colloidal structure that has a yield value upon deformation, and it should be reversible when subjected to shear, i.e., the primary modification of the parent liquid should be in a gel state that undergoes thinning when subjected to shear force. The great advantages of gels are the fact that they combine the properties of both liquid and solid.

Gels are non-Newtonian fluids, and their rheological properties play a vital role in quality control during production, and subsequent effective usage. Hence, rheological characterization is an essential step for better understanding of a gel system, and serves multiple purposes.
Such characterization helps to determine the apparent viscosity at different shear rates, for various gellants and operating ambient temperatures, and also to understand a gel's response to deformation when sheared. The knowledge obtained is essential in developing critical specifications for handling and transportation applications, and in determining the optimum conditions for the efficient injection of gel into a rocket engine system. They also help in the ability to control thrust, by controlling the mass flow rate of propellant in the combustion chamber; while ease of handling, and of storage, also reduce the risk of leakage. The clear understanding of fluid flow characteristics is vital for efficient combustion, in order to gain optimum performance. As a result, a significant effort has been made worldwide to formulate, prepare, and study the rheological properties of gel propellants for various applications in rocket propulsion systems [1-7]. In the current study, hydrogen peroxide based gel is examined and discussed, because it is eco-friendly. Hydrogen peroxide
This is an Open Access article distributed under the terms of the Creative Commons Attribution Non-Commercial License (http://creativecommons.org/licenses/by$\mathrm{nc} / 3.0 /$ ) which permits unrestricted non-commercial use, distribution, and reproduction in any medium, provided the original work is properly cited. (c) * Ph. D Student

** Professor, Corresponding author : swbaek@kaist.ac.kr 
serves as an oxidizing agent in the combustion of several organic fuels, and also serves as a monopropellant. The aims of the present study were to formulate propellant grade hydrogen peroxide gel, and to perform rheological characterization, using fumed silica $\left(\mathrm{SiO}_{2}\right)$ as gellant. The effect of low and high shear rates, and variation of ambient temperature on the rheological properties of hydrogen peroxide- $\mathrm{SiO}_{2}$ gelled system were also studied, to compare their relative suitability.

\section{Materials and Methods}

\subsection{Preparation of Gel Hydrogen peroxide}

Propellant grade hydrogen peroxide has been used as a bipropellant or monopropellant in many liquid rocket propulsion systems. Propellant grade hydrogen peroxide $\left(\mathrm{H}_{2} \mathrm{O}_{2}, 90 \%\right)$ was selected as a base propellant. The critical concentration of gellant required to gel hydrogen peroxide was determined by conducting several sets of experiments, between the range of 1 to $10 \mathrm{wt} \%$ of gellant. The gelling agent used for the gelation process has been found to be compatible and non-reactive with hydrogen peroxide. The gelation process is known to be affected not only by the type of gelling agent, but also by other parameters, such as gelation time, operating temperature, and particle size. Fumed Silica $\left(\mathrm{SiO}_{2}, 0.2 \sim 0.3 \mu \mathrm{m}\right.$ aggregate, Sigma Aldrich Corporation) (6 wt \%) was used in the present experiment for the gelation of hydrogen peroxide.

Fumed Silica $\left(\mathrm{SiO}_{2}\right)$ is a colloidal particulate, which is an amorphous form of silicon dioxide prepared by flame hydrolysis. Fumed Silica forms a weak hydrogen bonding, depending upon the type of solvent. In such a case, silanols on adjacent silica particles are envisioned to directly interact by weak hydrogen bonding. This leads to particle flocculation through a three-dimensional network of particles, and ultimately to gelation [8].

Dispersion of $\mathrm{SiO}_{2}$ particles in $\mathrm{H}_{2} \mathrm{O}_{2}$ liquid was prepared by thoroughly mixing them in a $50 \mathrm{ml}$ container of $40 \mathrm{~mm}$ I.D, at $1000 \mathrm{rpm}$ (3 blade laboratory Scale impeller) for 5 minutes. The suspension was stirred at room temperature $(298.15 \mathrm{~K}$ $\pm 1 \mathrm{~K})$, to ensure complete dissolution of the gellant in hydrogen peroxide. The mixture was then kept undisturbed, to allow network formation. In the hydrogen peroxide case, stable gel could be prepared. The main driving force for silica gelation is expected to be the formation of weak hydrogen bond between silanol $(\mathrm{Si}-\mathrm{OH})$ groups on adjacent silica units. The quality of the gel network obtained is usually dependent on the nature and purity of the liquid, grade, concentration, degree of dispersion of the gelling agent, type, and temperature involved. Uniformity in these critical aspects of the gelation process was maintained during all experimentations in this study.

During the process of gelation, the initial viscous liquid is converted into a self-supporting material, which exhibits viscoelastic characteristics. $\mathrm{H}_{2} \mathrm{O}_{2}$ gel with $\mathrm{SiO}_{2}$ shows thixotropic behavior. A detailed discussion is provided in the later section of this paper.

\subsection{Rheological Characterization}

Rheological study of freshly prepared $\mathrm{H}_{2} \mathrm{O}_{2}$ gel was carried out using a rotational rheometer (HAAKE RS600), and gel viscosity was assessed at the shear rate ranges of 1 to $20 \mathrm{~s}^{-1}$, and 1 to $1000 \mathrm{~s}^{-1}$. The low shear rate exercise is important to understanding the flow properties and minimum stress required for initiating flow (yield stress) of the gel at low shear rate, while ensuring uniform quality of the gel. On the other hand, the high shear rate exercise is most important from the rocket propulsion application point of view. A set of parallel plate geometry was used for the present investigation, since it is good for non-Newtonian linear viscoelastic fluids. A temperature control system inside the equipment ensured a constant operating temperature during all rheological measurements. The measurements were carried out at pre-selected temperatures $(283.15,293.15$ and $303.15 \mathrm{~K})$. The device used in this study allows the use of some parameter/conditions to carry out a measurement. For example, the apparent viscosity can be measured at different shear rates, at various ambient test temperatures, and for diverse "sample periods", i.e., the period of time, for which the shear rate is applied to the sample to get a single point. The equipment allows choice of how many points to get from the range of shear rate, sample period, and ambient temperature. If the fluid is time dependent, those parameters may affect the measurement data and its analysis. In those cases, a more detailed measurement procedure (listing the sampling period, number of experimental points, and steady ambient test temperature of sample) would be more appropriate, to make the data more precise, reproducible, and consistent. The viscosity measurement for $6 \mathrm{wt} \%$ gelled $\mathrm{H}_{2} \mathrm{O}_{2}$ was performed at a sampling time of $30 \mathrm{~s}$, and the total number of measurements was 100. In this experiment, the ambient test temperature of the device was preselected, and maintained. Subsequently, the gel was kept in a water bath for long enough time to attain the desired temperature. This sample was then placed on the device, while checking the gel steady test temperature again, before the apparent viscosity measurements were performed. As a result, we were able to 
obtain consistent and reproducible data for each condition. The sensor sensitivity of the device was approximately 0.01 ${ }^{\circ} \mathrm{C}$. The experimental study of the thixotropic character of hydrogen peroxide gel was also carried out by systematically increasing the shear rate, to record the up-curve of the rheogram; and then the shear rate was reduced in similar steps, to capture the down curve.

Non-Newtonian fluids, like gels, exhibit a complex flow behavior, unlike conventional fluids that have a predictable variation in their flow properties with shear rate and temperature.

\section{Results and Discussion}

In rheological study, the response of the freshly prepared $\mathrm{H}_{2} \mathrm{O}_{2}$ gel is measured under flow conditions, and the apparent viscosity is determined as a function of the shear rate. This study was conducted to investigate the time dependent shear-thinning (thixotropic) properties of hydrogen peroxide based gel in the 1 to $20 \mathrm{~s}^{-1}$, and 1 to $1000 \mathrm{~s}^{-1}$ shear rate regions. The measurement of rheological properties provides a better understanding of consistency, homogeneity, and the overall quality of gels. Critical information can also be gathered about the salient feature of micro-structure deformation, for fundamental characterization of the fluid behavior.

\subsection{Appearance}

Figure 1 depicts the hydrogen peroxide based gel with $6 \mathrm{wt}$ $\%$ of $\mathrm{SiO}_{2}$ gellant concentration. The much clearer and stiffer characteristics, and semi-solid like behavior of gel are clearly visible. Moreover, the samples appear to be homogenous, transparent, or slightly translucent gel, with no evidence of phase separation. Even visual examination indicates that the gel is a soft, freestanding solid that can bear its own weight in a sample container.

Hence, this class of materials could be categorized as $\mathrm{H}_{2} \mathrm{O}_{2}-\mathrm{SiO}_{2}$ gel.

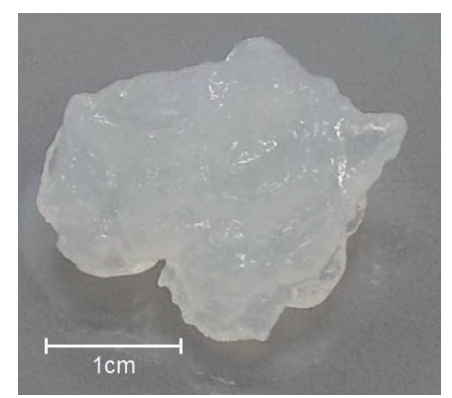

Fig. 1. Hydrogen peroxide gel with 6 wt $\%$ of $\mathrm{SiO}_{2}$ gellant

\subsection{Determination of Apparent Viscosity}

Shear rheology measurement is used to determine the flow properties, by changing the shear condition. Figs. 2 and 3 show the apparent viscosity as a function of the shear rate. The apparent viscosity in the current experiment for freshly prepared $\mathrm{H}_{2} \mathrm{O}_{2}-\mathrm{SiO}_{2}$ gel was determined in the 1 to 20 $\mathrm{s}^{-1}$, and 1 to $1000 \mathrm{~s}^{-1}$ shear rate ranges, for different ambient temperatures (283.15, 293.15, and 303.15 K).

The results on the variation of apparent viscosity in the 1 to $20 \mathrm{~s}^{-1}$ and 1 to $1000 \mathrm{~s}^{-1}$ shear rate ranges at different ambient temperatures $(283.15,293.15$ and $303.15 \mathrm{~K}$ ) for $\mathrm{H}_{2} \mathrm{O}_{2}-\mathrm{SiO}_{2}$ gel are presented in Figs. 2 and 3. The results clearly indicate that the apparent viscosity for gelled $\mathrm{H}_{2} \mathrm{O}_{2}$ significantly decreases with increasing shear rate. In the case of varying temperature and 1 to $20 \mathrm{~s}^{-1}$ shear rate range, the gel apparent viscosity not only decreases significantly, but the whole viscosity profile also shifts to the lower viscosity region. This could be due to the energy obtained with increase in temperature to overcome the resistance

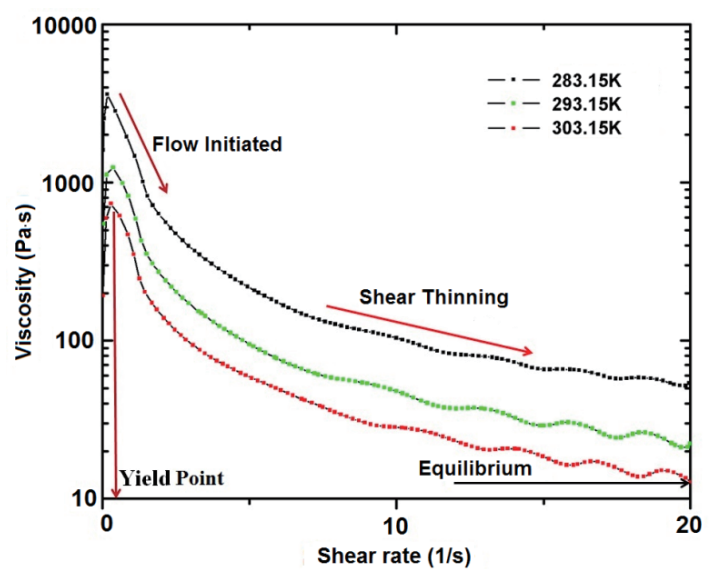

Fig. 2. Variation of the apparent viscosity of hydrogen peroxide gel in the 1 to $20 \mathrm{~s}^{-1}$ shear rate range, at ambient test temperature

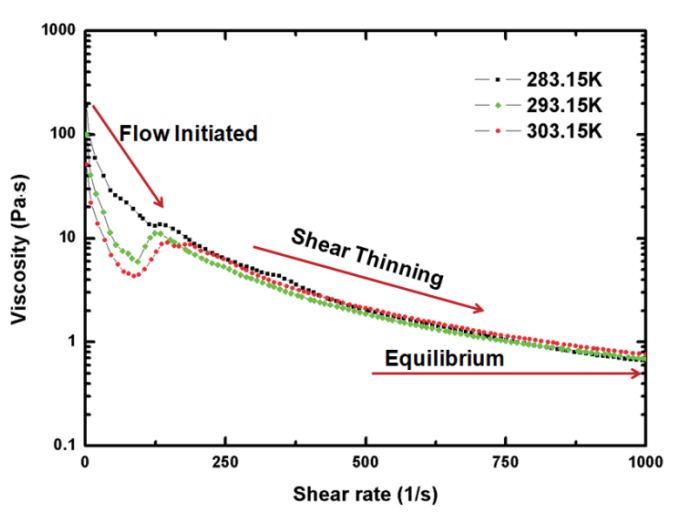

Fig. 3. Variation of the apparent viscosity of hydrogen peroxide gel in the 1 to $1000 \mathrm{~s}^{-1}$ shear rate range, at ambient test temperature 
to flow. This observation can be sustained by the fact that with increase in shear rate and temperature, the weaker inter-particle hydrogen bond that forms three-dimensional gel networks becomes further weakened. This may lead to a new alignment of network elements, hence allowing them to easily slip over one another, also resulting in the release of the entrapped liquid within, and offering less resistance to flow, so that there is reduced apparent viscosity. The results showing the effect of temperature on apparent viscosity variation at the shear rate range of 1 to $20 \mathrm{~s}^{-1}$ covered in the present experiment clearly show a substantial decrease in gel apparent viscosity with ambient temperature. However, in the shear rate range of 1 to $1000 \mathrm{~s}-1$, the gel apparent viscosity decreases as the shear rate increases, but no significant effect of varying the ambient temperature on the apparent viscosity was observed.

Figure 2 and 3 show that as soon as the $\mathrm{H}_{2} \mathrm{O}_{2}-\mathrm{SiO}_{2}$ gel is disturbed at a very low shear rate (less than $2 \mathrm{~s}^{-1}$ ), the flow is initiated with some yield stress [9] (in the case of the 1 to 20 s-1 shear rate range (Table 3)), and a further increase in shear rate leads to thinning of gel. The initial apparent viscosity of gel with some yield stress for $\mathrm{H}_{2} \mathrm{O}_{2}-\mathrm{SiO}_{2}$ gel decreases with increase in test temperature (the curve shifts downwards), which is possibly due to rapid breaking or weakening of gel network, at elevated temperatures from 283.15 to 303.15 K (Fig. 2). Absence of yield stress, and no significant effect of test temperature on the apparent viscosity of gel can be observed in the case of the 1 to $1000 \mathrm{~s}^{-1}$ shear rate range (Fig. 3). In general, it is also seen that the apparent viscosity linearly decreases with the shear rate, indicating that under the shear rate covered in the present study, the gel exhibits

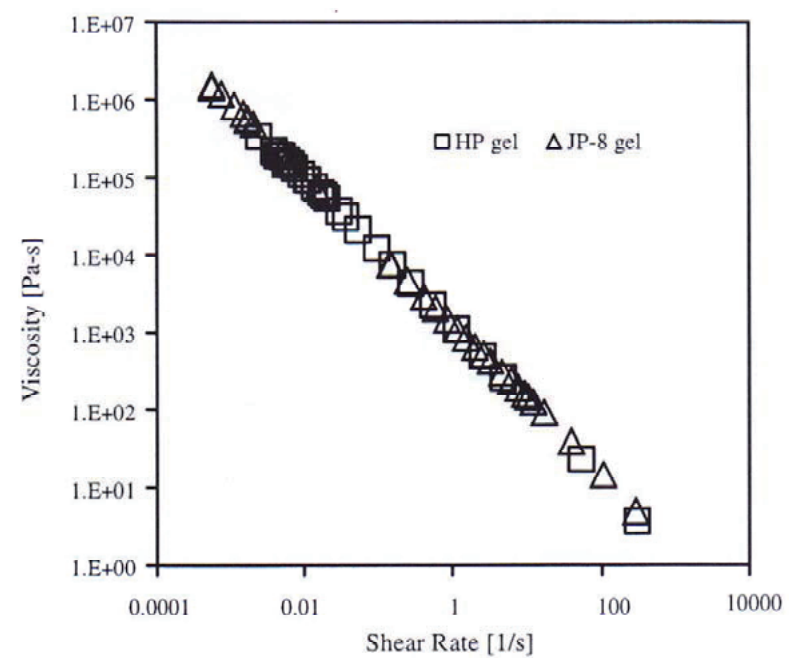

Fig. 4. The apparent viscosity of hydrogen peroxide gel in the 1 to $10000 \mathrm{~s}^{-1}$ shear rate range (rheological matching of a fuel gel and an oxidizer gel [7]) a pseudo-plastic character. This further establishes that the formulated hydrogen peroxide gel oxidizer has shearthinning and pseudo-plastic characteristics. Also, for the 1 to $20 \mathrm{~s}^{-1}$ and 1 to $1000 \mathrm{~s}^{-1}$ shear rate ranges, all the gel samples behave the same at one end of the flow curve; whereas, they show a dramatic difference at the other end, which is related to structural difference in these gels. The flow curve profiles for hydrogen peroxide gel in Fig. 2 and 3 show a similar shear thinning trend with increase in the shear rate for the shear rate range 1 to 1000 1/s, to the reference Figure 4 [7]. The gelation of hydrogen peroxide [7] (Figure 4) was done using fine powder silica with a standard tapped density of approximately $50 \mathrm{~g} / \mathrm{l}$ as a gelling agent, in a concentration ranging between 5 to $7 \mathrm{wt} \%[6,7]$, and it has a shear apparent viscosity range between 107 Pa.s and 100 Pa.s.

Summaries of the results obtained for the apparent viscosity and yield stress of $\mathrm{H}_{2} \mathrm{O}_{2}-\mathrm{SiO}_{2}$ gel for various temperatures are presented in Tables 1,2, and 3. The data clearly show that the initial viscosity of the hydrogen peroxide gel was very high at lower temperature, and then ultimately considerably decreased at higher temperatures at shear rates ranging from 1 to $20 \mathrm{~s}^{-1}$ and 1 to $1000 \mathrm{~s}^{-1}$. It is evident that the gel starts flowing at the nominal shear rate range of 1 to $20 \mathrm{~s}^{-1}$, with considerable reduction in apparent viscosity, with some yield stress, as given in Table 3 . This yield stress shows that the gel will not flow, unless it has reached a 'critical stress level' of so-called yield stress, i.e. the stress

Table 1. $\mathrm{H}_{2} \mathrm{O}_{2}$ gel apparent viscosity at a shear rate of 1 to $20 \mathrm{~s}^{-1}$

\begin{tabular}{cll}
\hline Temperature $(\mathrm{K})$ & $\eta_{\text {initial(Pa.s) }}$ & $\eta_{\text {final(Pa.s) }}$ \\
\hline 283.15 & 3614 & 53.8 \\
293.15 & 1246 & 22.3 \\
303.15 & 735 & 12.8 \\
\hline
\end{tabular}

Table 2. $\mathrm{H}_{2} \mathrm{O}_{2}$ gel apparent viscosity at a shear rate of 1 to $1000 \mathrm{~s}^{-1}$

\begin{tabular}{cll}
\hline Temperature $(\mathrm{K})$ & $\eta_{\text {initial(Pa.s) }}$ & $\eta_{\text {final(Pa.s) }}$ \\
\hline 283.15 & 189.2 & 0.67 \\
293.15 & 100.2 & 0.69 \\
303.15 & 51.3 & 0.76 \\
\hline
\end{tabular}

Table 3. $\mathrm{H}_{2} \mathrm{O}_{2}$ gel yield stress at a shear rate of 1 to $20 \mathrm{~s}^{-1}$

\begin{tabular}{cc}
\hline Temperature $(\mathrm{K})$ & $\sigma_{\mathrm{y}}(\mathrm{Pa})$ \\
\hline 283.15 & 590 \\
293.15 & 441.2 \\
303.15 & 210.5 \\
\hline
\end{tabular}


at which a material begins to deform. While below yield stress, $\mathrm{H}_{2} \mathrm{O}_{2}-\mathrm{SiO}_{2}$ gel is fully elastic; above it, a breakdown of the three-dimensional network structure of gel takes place, while starting to flow.

Shear-thinning pseudo-plastic behavior is very important, to ensure that with increase in shear rate to higher levels, the viscosity of the fluid would drastically decrease, to facilitate flow through injectors. This provides for safe handling and transportation. Presence of yield stress helps to avoid sedimentation, coagulation, and phase separation, and also helps in structural retention.

\subsection{Assessment of Thixotropic Behavior}

One of the ways of measuring thixotropy is to perform a hysteresis loop test [10]. In this study, the thixotropic [10] characteristics, along with the hysteresis loop behavior of freshly prepared hydrogen peroxide gel, were studied in the 1 to $20 \mathrm{~s}^{-1}$ and 1 to $1000 \mathrm{~s}^{-1}$ shear rate range, for different ambient temperatures $(283.15,293.15$, and $303.15 \mathrm{~K})$. The shear rate was systematically increased to a maximum level, to trace the up-curve; and then it was decreased in similar steps, to get the down-curve of the rheogram (Figs. 5 and 6 ). The data reveal that the apparent viscosity of the gel decreases significantly with shear rate; and because the shear stress values do not exhibit proportionality with the shear rate, the up and down curves cannot be superimposed on each other. Instead, they enclose an area between them, which is referred to as a 'hysteresis loop'. The area within the hysteresis loop provides a direct measure of the extent of the thixotropic load phase, the rapidity, and the completeness in temporal structural regeneration with subsequent decrease in shear rate, due to a local spatial rearrangement of the molecules in the three-dimensional network. The presence of the 'hysteresis loop' indicates

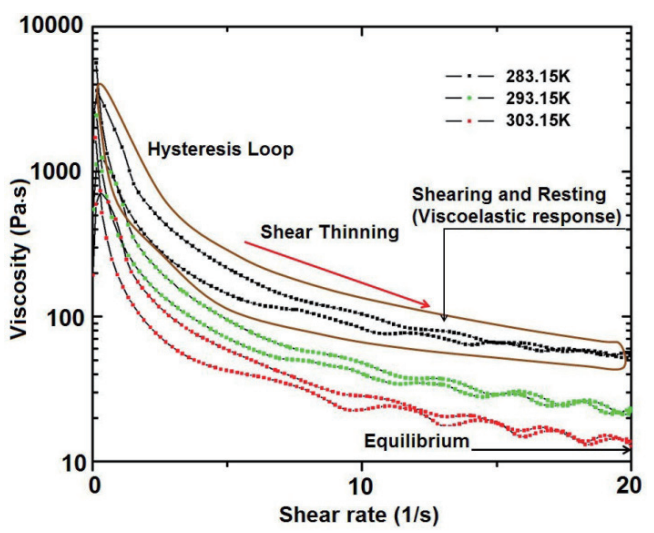

Fig. 5. Rheological test for thixotropic study of hydrogen peroxide gel for various ambient temperatures, at a shear rate of 1 to $20 \mathrm{~s}^{-1}$ that a breakdown of structure has occurred, and the area within the loop may be used as an index of the degree of breakdown, while representing the energy consumed in structural breakdown. The same phenomenon can be observed for all temperatures in Figs. 5 and 6. This indicates that the gel exhibits a thixotropic characteristic. The results were also analyzed using power law. The results show that the hydrogen peroxide gel exhibits a high shear-thinning property, and the thixotropic index, i.e., a measure of fluid flow property, is found to be more or less unaffected at higher temperature, for both the 1 to $20 \mathrm{~s}^{-1}$ and 1 to 1000 $\mathrm{s}^{-1}$ shear rate ranges. Hydrogen peroxide gel, when sheared, shows a larger departure from unity, indicating that much higher shear rates are required, to facilitate free flow for this gel (Table 4).

Figure 5 clearly reveals that, with increasing operating temperature, the apparent viscosity (ramp up- and downcurves) profile of the gel shifts to the lower viscosity region, in the 1 to $20 \mathrm{~s}^{-1}$ shear rate range. This can be attributed to the weakening of structural strength, which in turn is responsible for the reduction of gel viscosity at elevated temperature (i.e. the energy obtained to overcome the resistance to flow with increase in temperature). However, no significant effect of test temperature has been observed in the case of the 1 to $1000 \mathrm{~s}^{-1}$ shear rate range (Fig. 6). It is interesting to note that the hysteresis loop is just marginally affected by the variation in operating temperature in the 1 to $20 \mathrm{~s}^{-1}$, and 1

Table 4. $\mathrm{H}_{2} \mathrm{O}_{2}$ gel thixotropic index at shear rates of 1 to $20 \mathrm{~s}^{-1}$ and 1 to $1000 \mathrm{~s}^{-1}$

\begin{tabular}{cll}
\hline Temperature $(\mathrm{K})$ & $1-20(1 / \mathrm{s})$ & $1-1000(1 / \mathrm{s})$ \\
\hline 283.15 & -0.13 & -0.54 \\
293.15 & -0.09 & -0.38 \\
303.15 & -0.03 & -0.48 \\
\hline
\end{tabular}

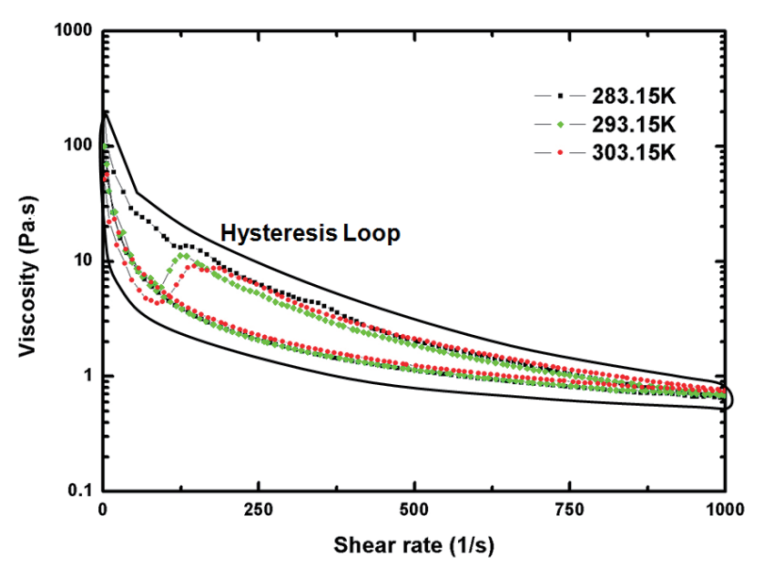

Fig. 6. Rheological test for thixotropic study of hydrogen peroxide gel for various ambient temperatures, at a shear rate of 1 to $1000 \mathrm{~s}^{-1}$ 
to $1000 \mathrm{~s}^{-1}$ shear rate ranges. However, gel in the 1 to $20 \mathrm{~s}^{-1}$ shear rate range does not lead to a complete breakdown of gel structure, in comparison to that in the 1 to $1000 \mathrm{~s}^{-1}$ range shear rate. The breakdown obviously became more pronounced at high shear rate, and the re-gelation process of the gels is also found to be much slower, when subjected to higher shear rates.

\section{Conclusion}

In the present study, gelation of propellant grade hydrogen peroxide was successfully performed with fumed silica as gelling agent at reasonably low critical concentration, to get $\mathrm{H}_{2} \mathrm{O}_{2}$ gel. The influence of the 1 to $20 \mathrm{~s}^{-1}$ and 1 to $1000 \mathrm{~s}^{-1}$ shear rate ranges for various operating temperatures led to a reduction in the apparent viscosity, with some yield stress (in the case of the 1 to $20 \mathrm{~s}^{-1}$ shear rate). Analysis of the results reveals that the gel exhibits a shear-thinning and thixotropic nature. The apparent viscosity and yield stress of hydrogen peroxide gel decreased with increase in shear rate, at higher temperature. The magnitude of apparent viscosity shifted to a much lower viscosity range, with increase in operating temperature, for gel system in the 1 to $20 \mathrm{~s}^{-1}$ shear rate range. No significant effect of different ambient temperature on the apparent viscosity was observed in the case of the 1 to 1000 $\mathrm{s}^{-1}$ shear rate. No significant change in the hysteresis loop and thixotropic index of gel was observed for different ambient temperature, for both the 1 to $20 \mathrm{~s}^{-1}$ and 1 to $1000 \mathrm{~s}^{-1}$ shear rates. The gel in the 1 to $20 \mathrm{~s}^{-1}$ shear rate does not lead to a complete breakdown of gel structure, in comparison to that in the 1 to $1000 \mathrm{~s}^{-1}$ shear rate. This shows that the operating temperature and shear rate together play an important role, and significantly influence the rheological properties of the $\mathrm{H}_{2} \mathrm{O}_{2}$ gel system.

\section{Acknowledgement}

This work was supported by grant No. EEWS-
2013-N01130030 from the EEWS Research Project of the office of KAIST EEWS Initiative (EEWS: Energy, Environment, Water and Sustainability).

\section{References}

[1] Munjal, N.L., Gupta, B.L., and Varma, M., "Preparative and Mechanistic Studies on Unsymmetrical Dimethyl Hydrazine-Red Fuming Nitric Acid Liquid Propellant Gels", Propellants, Explosives, Pyrotechnics, Vol. 10, 1985, pp. 111117.

[2] Varma, M., Gupta, B.L., and Pandey, M., "Formulation and Storage Studies on Hydrazine-Based Gelled Propellants", Defence Science Journal, Vol. 46, 1996, pp. 435-442.

[3] Gupta, B.L., Varma, M., and Munjal, N.L., "Rheological Studies on Virgin and Metallized Unsymmetrical Dimethyl Hydrazine Gelled Systems", Propellants, Explosives, Pyrotechnics, Vol. 11, 1986, pp. 45-52.

[4] Teipel Ulrich, and Forter Barth Ulrich., "Rheological Behavior of Nitromethane Gelled with Nanoparticles", $J$ Propul Power, Vol. 21, 2005, pp. 40-43.

[5] Natan, B., and Rahimi, S., "The Status of Gel Propellants in Year 2000", International Journal of Energetic Materials and Chemical Propulsion, Vol. 5, 2002, pp. 172-194.

[6] Shai Rahimi, and Arie Peretz., "On Shear Rheology of Gel Propellants", Propellants, Explosives, Pyrotechnics, Vol. 32, 2007, pp. 165-174.

[7] Shai Rahimi, Arie Peretz, and Benveniste Natan., "Rheological Matching of Gel Propellants", Journal of Propulsion and Power, Vol. 26, 2010, pp. 376-378.

[8] Srinivasa, R., and Raghavan., "Rheology of Silica Dispersions in Organic Liquids: New Evidence for Solvation Forces Dictated by Hydrogen Bonding", Langmuir, Vol. 16, 2000, pp. 7920-7930.

[9] Walls, H.J., Brett Caines, S., Angelica M. Sanchez, and Saad A. Khan., "Yield Stress and Wall Slip Phenomena in Colloidal Silica Gels", J. Rheol., Vol. 47, 2003, pp. 847-868.

[10] Howard, A. Barnes., "Thixotropy - a review”, J. NonNewtonian Fluid Mech, Vol. 70, 1997, pp. 1-33. 